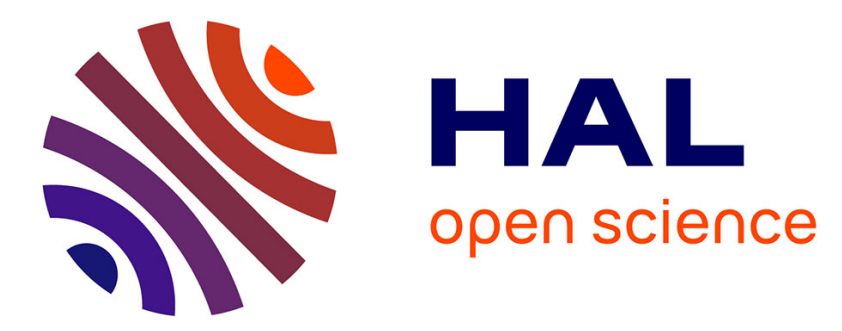

\title{
Psychopathologie quotidienne d'une institution à la lumière du cadre psychanalytique d'un groupe thérapeutique
}

\author{
Clarisse Vollon, P Drweski
}

\section{- To cite this version:}

Clarisse Vollon, P Drweski. Psychopathologie quotidienne d'une institution à la lumière du cadre psychanalytique d'un groupe thérapeutique. Revue de psychothérapie Psychanalytique de Groupes, 2018, 71, 10.3917/rppg.071.0037 . hal-02495708

\section{HAL Id: hal-02495708 \\ https://hal-amu.archives-ouvertes.fr/hal-02495708}

Submitted on 2 Mar 2020

HAL is a multi-disciplinary open access archive for the deposit and dissemination of scientific research documents, whether they are published or not. The documents may come from teaching and research institutions in France or abroad, or from public or private research centers.
L'archive ouverte pluridisciplinaire HAL, est destinée au dépôt et à la diffusion de documents scientifiques de niveau recherche, publiés ou non, émanant des établissements d'enseignement et de recherche français ou étrangers, des laboratoires publics ou privés. 


\section{Psychopathologie quotidienne d'une institution à la lumière du cadre psychanalytique d'un groupe thérapeutique}

C. VOLLON ${ }^{1}$, P. DRWESKI ${ }^{2}$

\section{Introduction}

Lorsque nous voulons mettre en place un groupe thérapeutique dans une institution il n'est pas rare que nous nous confrontions à des résistances de la part de l'équipe soignante. Lorsqu'elles ne sont pas énoncées explicitement, ces résistances peuvent se manifester de façon inconsciente sous forme d'attaques, de lapsus ou d'actes manqués: l'oubli d'aborder en réunion le projet du groupe thérapeutique, l'impossibilité pour les soignants de se rendre disponibles afin de participer au dispositif, la salle du groupe utilisée pour une autre activité au moment de la séance... Des résistances qui sont, souvent, l'expression de disfonctionnements et de souffrances institutionnelles. La construction et la mise en place du dispositif de groupe peut devenir alors laborieux, voir mis en échec. Ainsi, nous sommes amenés à penser un cadre thérapeutique qui permet non seulement d'aménager un espace d'élaboration et de soins pour les patients, mais également de contenir et traiter ces attaques institutionnelles. Dans cette perspective, nous souhaitons avancer dans cet article l'hypothèse que le cadre psychanalytique d'un groupe thérapeutique, dans sa forme et son évolution, offre un éclairage sur le fonctionnement psychique ordinaire de l'institution dans laquelle il s'organise.

\footnotetext{
1 VOLLON Clarisse, docteur en psychologie clinique, psychologue clinicienne, attachée temporaire d'enseignements et de recherches Université Paris Descartes, PCPP EA4056, Sorbonne Paris Cité, France. vollonclarisse@gmail.com.
}

${ }^{2}$ DRWESKI Philippe, docteur en psychologie clinique, psychologue clinicien, membre temporaire du laboratoire PCPP EA4056, Sorbonne Paris Cité, France.philippe.drweski@gmail.com 


\section{Rappels autour de la notion de cadre}

Le mot "cadre " provient du latin quadrum et signifie carré. II peut définir soit l'ensemble de châssis qui entourent un objet à exposer ou à tenir, soit l'ensemble des gradés d'une troupe militaire. En tauromachie, le mot "cadre " renvoie à l'immobilisation de l'animal par un ensemble de passes avant de l'estoquer. Repérons ici que le mot cadre est avant tout un moyen pour atteindre un objectif : il est question d'ordre et de maîtrise. Ainsi, le cadre thérapeutique s'appréhende dans le mouvement et la dualité : il se caractérise à la fois par ce qu'il est mais aussi parce qu'il permet de faire. Nous savons que Winnicott, en 1971, parle de Setting pour définir la notion de cadre, c'est-à-dire l'ensemble de tous les détails concernant la conduite du thérapeute ainsi que de l'aménagement du dispositif. Le cadre permet alors la création d'une aire intermédiaire, un espace entre deux où patient et soignant vont pouvoir se rencontrer. Ce cadre peut être aussi envisagé dans une dimension polysémique comme le propose Green (1995), composé d'une partie matricielle formée par le couple thérapeute/ patient qui contient une partie écrin qui va être le lieu du transfert, en d'autres termes là où la rencontre va pouvoir être possible. Enfin, Bleger (1967) envisage le cadre comme une institution, à l'intérieure de laquelle se produit un certain nombre de comportements. Ce cadre ne peut pas être perçu, ou sauf lorsqu'il se modifie ou se casse, provoquant alors une « angoisse catastrophique " (Fustier, 2008). C'est l'implicite dont dépend l'explicite, Bleger évoque pour nommer le cadre, les notions de «monde fantôme " ou de "méta-comportement". Ainsi, la construction du cadre s'articule non seulement autour d'un certain nombre de constantes comme des repères spatio-temporels mais également d'une partie de la personnalité du patient et de son thérapeute. Cette personnalité correspond à la partie la plus primitive, la plus régressée de notre personnalité totale et qui fonctionne 
encore sur les modalités de la symbiose mère-bébé. Kaës $(1979,1994)$ a repéré plusieurs fonctions du cadre. Tout d'abord, le cadre a une fonction contenante. Il permet en effet de contenir les parties psychotiques de la personnalité (Bion, 1961), des affects, des représentations du corps voir des objets internes. Le cadre a également une fonction limitante : il garantit les limites psychiques du sujet, et permet la constitution d'une intériorité et d'une extériorité corporelle et psychique. Par ailleurs, le cadre a une fonction symboligène, il est condition de la pensée grâce au processus de symbolisation qu'il instaure. Enfin, le cadre a une fonction transitionnelle telle que Winnicott avait déjà pu envisager cette notion en 1971. Quelles sont alors les spécificités du cadre des groupes thérapeutiques?

\section{Le cadre dans les groupes thérapeutiques : entre transitionnalité, créativité et parts syncrétiques des personnalités}

Le groupe thérapeutique permet l'expérience pour ses membres du passage de l'omnipotence à l'illusion, la possibilité de symboliser via la mise en place d'une enveloppe collective (Privat, 2007). La fonction limitante de son cadre, mais aussi d'échangeur entre le dedans et le dehors aménage une aire transitionnelle, où pourra s'élaborer une activité groupale de pensée (Ibid). Plus spécifiquement, le choix du cadre des groupes thérapeutiques est fonction des hypothèses de travail dans ces dispositifs : celles qui traitent des individus en groupe sans tenir compte des phénomènes de groupe, celles qui reconnaissent le groupe comme une réalité psychologique spécifique, et celles enfin qui prennent en compte les effets de groupe sur les effets thérapeutiques individuels (Quélin-Sélignoux, 2007). Dans cette perspective, trois types de groupes thérapeutiques peuvent être instaurés: soit des psychothérapies de groupe où la finalité psychothérapeutique est en elle-même l'objet de travail 
du groupe, soit des groupes à médiations, où c'est la relation à la médiation qui est essentielle dans le groupe, soit enfin des groupes thérapeutiques médiatisés (l'expérience partagée autour de la proposition de médiation permet de travailler à un changement). Le choix du lieu des séances est aussi tout à fait fondamental. Véritable espace de création (Duchet, 2016), le cadre du groupe est également le support de l'activité fantasmatique des membres du groupe et mettra en jeu la dynamique des frontières, la désignation de places, la figuration des désirs, l'économie pulsionnelle (Douarche, 2012, p. 84). En effet, la base matérielle du groupe est l'espace qu'il trouve et qu'il crée et par extension l'assise d'un groupe se présente comme un territoire (Kaës, 1976).

Seulement, comment penser la construction du cadre thérapeutique au regard des spécificités de la dynamique et du fonctionnement de l'institution dans lequel le groupe se déroule ? Nous savons que le cadre d'un groupe s'étaye sur le cadre institutionnel ce qui conduit à un emboîtement du groupe psychothérapeutique et du groupe institution, pouvant entraîner par ailleurs un parasitage inter cadres (Kaës, 1979). Or, Selon Bleger (1989), les institutions sont dépositaires de la "sociabilité syncrétique ", c'est-à-dire " l'immobilisation de l'individuation et de la non-relation ". Puisque le cadre d'un groupe thérapeutique s'étaye sur le cadre institutionnel, il est également dépositaire de cette sociabilité, en plus de la part syncrétique de la personnalité du thérapeute et des patients qui composent le groupe. L'illustration clinique qui va suivre va nous permettre d'observer que c'est justement l'expression de ces différentes parts syncrétiques fondatrices du cadre thérapeutique du groupe qui nous permettent d'avoir accès au fonctionnement psychique ordinaire de l'institution. 


\section{Illustration clinique}

Il s'agit d'un psychodrame psychanalytique de groupe monté au sein d'une structure ambulatoire. Ce groupe est co-animé par deux psychologues et s'adresse à des adolescents entre 12 et 15 ans. Ce dispositif existe dans l'institution depuis 4 ans et se déroule de façon hebdomadaire pendant une heure.

\section{Contexte et construction du cadre du groupe}

Un psychodrame individuel avait été mis en place quelques années auparavant dans cette même institution mais sans mobiliser l'intérêt de l'équipe. En effet, pendant les réunions de synthèses, le dispositif était très rarement mentionné et peu d'orientations thérapeutiques étaient proposées en dehors du psychologue qui l'animait. Les échanges autour de ce groupe étaient souvent informels, s'effectuant dans des espaces interstitiels ( $R$. Roussillon, 1987) révélant alors un manque d'ancrage institutionnel du dispositif. Du fait de la difficulté de l'équipe à pouvoir porter ce projet, ce psychodrame individuel fût arrêté par le psychologue. Deux ans plus tard, le désir de remettre en place un nouveau psychodrame a émergé, désir porté par deux cliniciens du centre. II faisait suite à une réflexion concernant des patients en difficulté pour élaborer leurs propres mouvements psychiques. À cette époque le médecin directeur du centre était régulièrement absent pendant de longues périodes. Pourtant, les prémices de la construction du cadre de ce groupe de psychodrame parvinrent à s'inscrire dans un travail d'élaboration, en amont, avec l'institution. Il fut décidé de reprendre dans ce dispositif les règles classiques du psychodrame (liberté de parole, confidentialité, faire semblant...) mais aussi de penser la place des parents. Dans cette perspective, il fut proposé 
de rencontrer les parents et les patients afin de "faire le point " sur leur participation au dispositif. Cet espace respectait, bien entendu, la règle de la confidentialité du groupe. Les indications thérapeutiques furent également précisées et reprises en équipe : un psychodrame pour des adolescents avec des difficultés de représentations de leurs mouvements psychiques. Cela pouvait ainsi concerner des pathologies diverses de la psychose à la névrose et pour lesquelles la figuration du psychodrame apportait un étayage particulièrement propice au travail thérapeutique. Un dernier élément du dispositif devait donc être décidé avant de commencer le groupe : son lieu. Le centre n'ayant pas assez de bureau, il n'était pas possible d'avoir une salle spécialement dédiée aux différents groupes. Seul celui du médecin directeur avait la capacité d'accueillir l'ensemble des participants, et se fut dans cet espace que les premières séances s'effectuèrent.

\section{Mise en place du groupe et évolution institutionnelle}

Durant les premiers temps du groupe des attaques institutionnelles s'exprimèrent à travers des réflexions sur le dispositif, jamais énoncées lors des réunions de synthèse, mais plutôt " entre deux portes " comme : "Avant nous n'avions pas besoin de tout ça (en parlant des dispositifs de groupe) " ou bien "Si on fait trop de groupe il n'y aura plus de place pour les prises en charge individuelles » et encore "On fait du chiffre ». Echappant au moment de la synthèse, ces remarques pouvaient être appréhendées comme des formes de "lapsus institutionnels", révélant ainsi la pensée inconsciente de l'institution sur le groupe. En effet, ces phrases exprimaient, en négatif, une série de fantasmes soulevés par la mise en place des dispositifs de soins en groupe dans la structure. Entre nostalgie institutionnelle incarnée par l'idéalisation d'une période où il n'y avait pas besoin de groupe thérapeutiques 
dans la structure de soin et fantasmes d'intrusion, le groupe semblait représenter pour l'équipe une forme "moins pure" de thérapie. Enfin la question du chiffre délégitimait le psychodrame, l'incluant dans une logique comptable proche des préoccupations de la direction et dénigrée par les soignants.

Dans un même temps, le médecin directeur décida de partir sans qu'il soit possible pour l'équipe de lui dire au revoir. Un nouveau médecin directeur le remplaça rapidement et de nombreux conflits émergèrent entre lui et les autres soignants : les synthèses étaient marquées par des tensions croissantes, la parole circulait avec difficulté, la capacité de penser était attaquée.

\section{Attaques du dispositif de groupe}

Ces différents mouvements institutionnels eurent des effets très directs sur le dispositif. Malgré des indications posées lors des réunions de synthèse, de moins en moins d'adolescents participaient au psychodrame, au point qu'il n'y eu plus qu'un seul participant. Cette évolution du groupe faisait écho à la modification du fonctionnement institutionnel suite à l'arrivée du nouveau médecin directeur. Ce dernier imposa d'ailleurs rapidement de rencontrer l'ensemble des parents suivis dans la structure, conduisant à une confusion avec l'espace de rencontre parents/adolescents prévu dans le dispositif psychodramatique. Une situation clinique l'illustre. II s'agit de celle d'Adrien, 13 ans et de sa famille. Adrien est adressé pour des problèmes de comportements à l'école et à la maison. Ses parents se plaignent de son immaturité et de sa difficulté à s'approprier ses propres mouvements psychiques (colère, tristesse). Le projet thérapeutique élaboré en réunion puis proposé à cette famille est un suivi psychodramatique en groupe ainsi que des rendez-vous parentaux avec le médecin directeur. Le père du jeune garçon a très rapidement arrêté de se 
présenter au rendez-vous médicaux avançant qu'ils " ne servent à rien ». Le médecin interroge alors les rendez-vous du dispositif psychodramatique en posant la question d'un " télescopage " de ses rendez-vous et du dispositif de psychodrame. La représentation que les deux espaces viennent se confondre émerge, empêchant ainsi toute articulation du travail clinique. Les conséquences sur la prise en charge se font rapidement ressentir: Adrien s'absentera des séances du psychodrame sans pouvoir en dire quelque chose dans le groupe. Le fonctionnement institutionnel, sans doute en résonance avec le fonctionnement familial, ne permis pas d'accueillir et de travailler avec Adrien.

\section{Réflexions cliniques}

A partir de cette illustration, plusieurs réflexions cliniques se dégagent. Nous pouvons repérer la difficulté avec laquelle un groupe thérapeutique peut s'organiser dans la structure soignante (arrêt il y a plusieurs années d'un premier groupe de psychodrame, difficultés aujourd'hui d'en maintenir un second). Nous pensons qu'il s'agit ici de l'expression de résistances institutionnelles: plus spécifiquement l'expression de l'impossibilité pour le groupe soignant de porter psychiquement un groupe thérapeutique, de créer une place pour l'élaboration, d'accueillir et permettre un emboîtement des cadres (Kaës, 1976). Ces résistances s'expriment non seulement dans ses "lapsus institutionnels » mais aussi dans la construction et l'évolution du cadre thérapeutique du psychodrame actuel. En effet, nous repérons qu'il s'organise progressivement comme un psychodrame individuel: la diminution significative au fil des mois de l'adresse de patients par l'équipe dans ce groupe vient modifier structurellement le dispositif de soin au point d'en questionner in fine la dimension groupale. Par ailleurs, l'importance accordée à l'échange 
avec les parents des participants au groupe questionne également. Nous pouvons observer que cette composante du cadre du groupe de psychodrame se télescope voire rivalise avec certaines pratiques thérapeutiques dans l'institution, ici le travail imposé par le nouveau médecin directeur. Pourtant, le choix d'aménager cet espace, ce groupe en parallèle du dispositif psychodramatique, peut s'envisager comme une tentative de légitimer cette pratique thérapeutique, tout se passant comme si "faire le point " avec les parents permettait en creux, de faire le point avec cette institution mère qui peine à accepter qu'un groupe émerge et s'autonomise en son sein. Le cadre du groupe de psychodrame devient alors bien ce support de l'activité fantasmatique institutionnelle, la figuration de ses désirs et de son économie pulsionnelle (Douarche, 2012, p. 84). Mais nous observons aussi plus largement que le cadre thérapeutique d'un groupe constitue un double vertex (Bion, 1965, p. 106), c'est-à-dire un double équivalent mental permettant au thérapeute de se projeter dans deux espaces différents : ici celui du groupe de psychodrame et celui de l'institution. Dis autrement, le cadre thérapeutique du groupe offre un éclairage et une articulation entre deux réalités groupales, celle des patients et celle de la structure qui les accueille.

\section{En guise de conclusion}

Dans cette perspective et pour conclure, le cadre d'un groupe thérapeutique se construit non seulement à partir des aménagements voulu par le thérapeute mais également à partir des éléments inconscients qui circulent dans une institution. S'adossant à l'enveloppe collective qui borde la structure de soin, les spécificités de l'organisation du cadre d'un groupe thérapeutique constitue une porte d'accès à la compréhension du fonctionnement psychique ordinaire de l'institution. Ainsi, au-delà de ses 
aspects transitionnel, créatif et syncrétique, le cadre d'un groupe a une dimension biface : il porte les spécificités de la réalité psychique inconsciente institutionnelle et de la réalité psychique inconsciente du groupe qu'il accueille. De fait, le cadre doit nécessairement revêtir une qualité plastique pour pouvoir s'adapter non seulement aux besoins et demandes du groupe mais aussi aux résistances et exigences institutionnelles. Ce dernier point nous conduit à revenir sur les écrits d'André Green et les notions de matrice et d'écrin. Serait-il possible d'envisager la partie matricielle composée du thérapeute, des membres du groupe et des parts syncrétiques de l'institution et la partie écrin composée quant à elle des liens transférentiels groupaux mais également des liens transférentiels institutionnels ? Cette question soulève la nécessité, peutêtre, de reprendre ces notions pour mieux comprendre les enjeux d'une réflexion autour de la construction du cadre et la psychopathologie quotidienne du groupe institution. 


\section{Références bibliographiques}

BION, W.R. 1961. Recherche sur les petits groupes. Paris : Presses Universitaires de France. BION, W.R. 1965. Transformations, passage de l'apprentissage à la croissance. Paris : Presses Universitaires de France.

BLEGER, J. 1967. Symbiose et ambiguïté. Paris : Presses Universitaires de France.

BLEGER, J. 1989. « Le groupe comme institution et le groupe dans les institutions », in R. Kaës (dir.), L'institution les institutions (3 ${ }^{\text {ème }}$ éd., vol. 1, pp. 47-62). Paris : Dunod.

DOUARCHE, R. 2012. "De la vertue du cadre oublié ", in Revue de Psychothérapie Psychanalytique de Groupe, 59, pp. 83-94

DUCHET, C., KERSALÉ, M., ABRGALL G. 2016. «Le cadre en configuration groupale... un processus en création ?", in Revue de Psychothérapie Psychanalytique de Groupe, 66, pp. 161-172.

FUSTIER, P. 2008. Les corridors du quotidien. Paris : Dunod.

GREEN, A. 1995. La causalité psychique : entre nature et culture. Paris : Broché.

KAËS, R. 1976. L'appareil psychique groupal. Paris : Dunod.

KAËS , R. 1979. Crise, rupture et dépassement. Paris : Dunod.

KAËS, R. 1994. La parole et le lien. Paris : Dunod.

MORENO, J. 1954. Psychothérapie de groupe et psychodrame. Paris : Presses Universitaires de France.

PRIVAT, P., QUELIN SELIGNOUX, D., ROUCHY, J.-C. 2001. "Psychothérapie psychanalytique de groupe ", in Revue de Psychothérapie Psychanalytique de Groupe, 37, pp. 11-30

ROUSSILLON, R. 1999. Agonie, clivage et symbolisation. Paris : Presses Universitaires de France.

WEIL, M. 2012. "Réflexions sur les psychothérapies psychanalytiques de groupe en hôpital psychiatriques ", in Revue de Psychothérapie Psychanalytique de Groupe, 59, pp. 23-30.

WINNICOTT, D. 1971. Jeu et réalité. Paris : Folio Essais.

\section{Résumé}

Nous avançons dans cet article que la forme et l'évolution d'un cadre psychanalytique d'un groupe thérapeutique offre un éclairage sur le fonctionnement psychopathologique de l'institution dans laquelle il s'organise. Pour illustrer notre propos, nous reviendrons dans un premier temps sur la notion de cadre et plus spécifiquement la notion de cadre 
psychanalytique d'un groupe. Dans un second temps, à partir d'une situation clinique, un psychodrame psychanalytique de groupe mené dans un service de pédo-psychiatrie, nous serons conduits à envisager le cadre d'un groupe thérapeutique comme une construction s'appuyant sur les aménagements voulus par le thérapeute mais également sur les éléments inconscients qui circulent dans une institution. Le cadre psychanalytique d'un groupe thérapeutique présentera alors une dimension biface permettant d'avoir accès à deux réalités psychiques distincts: celles du groupe thérapeutique, et celle de l'institution qui l'accueille.

Mots clefs : institution, psychopathologie, groupe, psychodrame, cadre psychanalytique.

\begin{abstract}
We propose in this article that the form and evolution of a psychoanalytic framework of a therapeutic group offers insights into the psychopathological functioning of the institution in which it is organized. To illustrate our point, we will return first to the notion of framework and more specifically the concept of psychoanalytic framework of a group. In a second step, from a clinical situation, a group psychoanalytic psychodrama conducted in a child psychiatry service, we will be led to consider the framework of a therapeutic group as a construction based on the adjustments required by the therapist but also on the unconscious elements circulating in an institution. The psychoanalytic framework of a therapeutic group will then have a two-sided dimension allowing access to two distinct psychic realities: those of the therapeutic group, and that of the institution that hosts it.
\end{abstract}

Keywords: institution, psychopathology, group, psychodrama, setting. 